\title{
A SPECIAL INTEGRAL AND A GRONWALL INEQUALITY
}

\section{BY}

\section{BURRELL W. HELTON}

In memory of Professor R. L. Moore

ABSTRACT. This paper considers a special integral $(I) \int_{a}^{b}(f d g+H)$ which is a subdivision-refinement-type limit of the approximating sum

$$
\sum_{1}^{n}\left\{f\left(t_{i}\right)\left[g\left(x_{i}\right)-g\left(x_{i-1}\right)\right]+H\left(x_{i-1}, x_{i}\right)\right\},
$$

where $x_{i-1}<t_{i}<x_{i}$. The author shows, with appropriate restrictions, that (I) $\int_{a}^{b}(f d g+H)$ exists if and only if

$$
\text { (R) } \int_{x}^{y}\left(f d g+H-A^{-}\right)=(L) \int_{x}^{y}\left(f d g+H+A^{+}\right)
$$

for $a<x<y<b$, where $A(p, q)=[f(q)-f(p)][g(q)-g(p)], A^{-}(p, q)=$ $A\left(q^{-}, q\right)$ and $A^{+}(p, q)=A\left(p, p^{+}\right)$. Furthermore, if either of the equivalent statements is true, then all the integrals are equal. These equivalent statements are used to prove an integration-by-parts theorem and to solve a Gronwall inequality involving this special integral. Product integrals are used in the solution of the Gronwall inequality.

Introduction. This paper considers a special integral $(I) \int_{a}^{b}(f d g+H)$ which is a subdivision-refinement-type limit of the approximating sum

$$
\sum_{1}^{n}\left\{f\left(t_{i}\right)\left[g\left(x_{i}\right)-g\left(x_{i-1}\right)\right]+H\left(x_{i-1}, x_{i}\right)\right\},
$$

where $x_{i-1}<t_{i}<x_{i}$ for $i=1,2, \ldots, n$. All functions are from real numbers to real numbers. Since the function $H$ might be defined as $H(x, y)=$ $u(x)[r(y)-r(x)]+v(y)[s(y)-s(x)]$, then the Cauchy left and right integrals, the Smith mean integral [9], and the weighted integral of Wright and Baker [13] are special cases of this integral. We define $A, A^{-}$and $A^{+}$to be the functions $A(x, y)=[f(y)-f(x)][g(y)-g(x)], A^{-}(x, y)=A\left(y^{-}, y\right)$ and $A^{+}(x, y)=$ $A\left(x, x^{+}\right)$; then we show, with suitable restrictions, that

$$
(I) \int_{a}^{b}(f d g+H)
$$

Received by the editors February 27, 1973 and, in revised form, October 31, 1974.

AMS (MOS) subject classifications (1970). Primary 26A42, 26A86; Secondary 26A39, 28A25, 45A05.

Key words and phrases. Integrals, Gronwall's inequality, product integrals, integration-by-parts, bounded variation, Smith mean integral, Cauchy integrals, Dushkin interior integral, subdivision-refinement-limit. 
exists if and only if $(L) \int_{x}^{y}\left(f d g+H+A^{+}\right)=(R) \int_{x}^{y}\left(f d g+H-A^{-}\right)$for $a \leqslant x<$ $y \leqslant b$; furthermore, if either of the statements is true, then all the integrals are equal;

$$
\text { (I) } \int_{a}^{b}(f d g+H)=(I) \int_{a}^{b}(H-g d f)+f(b) g(b)-f(a) g(a)+\int_{a}^{b}\left(A^{+}-A^{-}\right) ;
$$

and

(3) there is a function $u$ such that, if $f(x) \leqslant h(x)+(L I R) \int_{a}^{x}(f H+f G+f K)$ for $a \leqslant x \leqslant b$, then $f(x) \leqslant u(x)$ for $a \leqslant x \leqslant b$; product integrals are used in defining the function $u$.

An integration-by-parts theorem of Wright and Baker [13] is a special case of Theorem 4. The Gronwall inequality theorems of Wright, Klasi and Kennebeck [14] and Helton [4] are special cases of Theorem 5. Some other recent developments pertaining to the Gronwall inequality may be found in the papers listed in the bibliography.

Definitions and notations. Each integral and product integral is a subdivisionrefinement-type limit and $R$ is the set of real numbers. The number set $\left\{x_{i}\right\}_{0}^{n}$ is a subdivision of $[a, b]$ and $\left\{t_{i}\right\}_{1}^{n}$ is an interpolating sequence for $\left\{x_{i}\right\}_{0}^{n}$ imply that $a=x_{0}<t_{1}<x_{1}<t_{2}<x_{2}<\cdots<x_{n}=b$.

An ordered set $\{L, I, R\}$ of letters preceding an integral symbol indicates the type of approximating sum for each term of the integral-Cauchy left integral $(L)$, Cauchy right integral $(R)$, interior (Dushnik) integral ( $I)$; for example,

$$
\begin{aligned}
&(L I R) \int_{a}^{b}(f d g+h G+H+g d x) \\
&=\sum_{1}^{n}\left\{f\left(x_{i-1}\right)\left[g\left(x_{i}\right)-g\left(x_{i-1}\right)\right]+h\left(t_{i}\right) G\left(x_{i-1}, x_{i}\right)\right. \\
&\left.+H\left(x_{i-1}, x_{i}\right)+g\left(x_{i}\right)\left(x_{i}-x_{i-1}\right)\right\}
\end{aligned}
$$

where $x_{i-1}<t_{i}<x_{i}$ for $i=1,2, \ldots, n$.

If $G$ is a function from $R \times R$ to $R$, then $G \in O A^{\circ}$ on $[a, b]$ means $\int_{a}^{b}\left|G-\int G\right|=0, G \in O M^{\circ}$ on $[a, b]$ means ${ }_{x} \Pi^{y}(1+G)$ exists for $a \leqslant x<y \leqslant b$ and $\int_{a}^{b}|(1+G)-\Pi(1+G)|=0$, and $G \in O L^{\circ}$ on $[a, b]$ means, if $a \leqslant x<y \leqslant b$, then $G\left(x, x^{+}\right), G\left(x^{+}, x^{+}\right), G\left(y^{-}, y\right)$ and $G\left(y^{-}, y^{-}\right)$exist. $G \in O B^{\circ}, G^{-1}$ exists and $G \geqslant c$ on $[a, b]$ means there is a number $M$ and a subdivision $D$ of $[a, b]$ such that if $\left\{x_{i}\right\}_{0}^{n}$ is a refinement of $D$, then $\Sigma_{1}^{n}\left|G\left(x_{i-1}, x_{i}\right)\right|<M,\left[G\left(x_{i-1}, x_{i}\right)\right]^{-1}$ exists for $0<i \leqslant n$, and $G\left(x_{i-1}, x_{i}\right) \geqslant c$ for $0<i \leqslant n$, respectively. (Where one or more of these properties are needed in a proof, we will assume that an appropriate subdivision has been introduced into the proof.) The symbol $d g$ represents a function $G$ such that $G(x, y)=g(y)-g(x)$. See [3] and [4] for 
detailed definitions. If the meaning is clear, the words "on $[a, b]$ " will be omitted and the symbols $f_{i-1}, f_{i}, G_{i}$, etc. will be used as shorthand symbols for $f\left(x_{i-1}\right)$, $f\left(x_{i}\right), G\left(x_{i-1}, x_{i}\right)$, etc.

The symbols $A, A^{+}$and $A^{-}$are used to denote functions from $R \times R$ to $R$ such that, if $a \leqslant x<y \leqslant b$, then $A(x, y)=[f(y)-f(x)][g(y)-g(x)]$, where $f$ and $g$ are functions from $R$ to $R$, and $A^{+}(x, y)=A\left(x, x^{+}\right)$and $A^{-}(x, y)=$ $A\left(y^{-}, y\right)$. Note that:

(1) if $d g \in O B^{\circ}$ and $f$ is bounded on $[a, b]$, then $A \in O B^{\circ}$ on $[a, b]$;

(2) if $A \in O B^{\circ}$ and $A^{+}$exists on $[a, b]$, then $\int_{a}^{b} A^{+}$exists; and

(3) if $\lim _{x \rightarrow p^{+}}[g(p)-g(x)] \neq 0$ and $A\left(p, p^{+}\right)$exists, then $f\left(p^{+}\right)$exists.

Main results. The lemmas mentioned in the following theorems are stated in the following section.

THEOREM 1. Given. $[a, b]$ is a number interval, $H$ is a function from $R \times R$ to $R$, and $f$ and $g$ are functions from $R$ to $R$ such that $A^{+}$and $A^{-}$exist and $A \in$ $O B^{\circ}$ on $[a, b]$ and such that, if $a \leqslant x<y \leqslant b$, then $\int_{x}^{y}\left(A-A^{+}-A^{-}\right)$exists and is zero, and

$$
\lim _{p, q \rightarrow x^{+}}[f(q)-f(p)][g(q)-g(x)]=0,
$$

and

$$
\lim _{p, q \rightarrow y^{-}}[f(q)-f(p)][g(q)-g(y)]=0
$$

Conclusion. If one of the following integrals exists, then the other integrals exist, and

$$
(L) \int_{a}^{b}\left(f d g+H+A^{+}\right)=(I) \int_{a}^{b}(f d g+H)=(R) \int_{a}^{b}\left(f d g+H-A^{-}\right) .
$$

Proof. Let $\epsilon>0$. Since $\int_{a}^{b}\left(A^{+}+A^{-}-A\right)$ exists, then $A^{+}+A^{-}-$ $A \in O A^{\circ}$. Since $A \in O B^{\circ}$ and $A^{+}$and $A^{-}$exist on $[a, b]$, then $\int_{x}^{y}\left(A^{+}+A^{-}\right)$ and $\int_{x}^{y} A$ exist and $\int_{x}^{y}\left(A^{+}+A^{-}\right)=\int_{x}^{y} A$ for $a \leqslant x<y \leqslant b$; hence, the hypothesis of Lemma 3 is satisfied. Therefore, there is a subdivision $D=\left\{z_{i}\right\}_{0}^{m}$ of $[a, b]$ such that if $D^{\prime}=\left\{x_{i}\right\}_{0}^{n}$ is a refinement of $D$ and $\left\{t_{i}\right\}_{1}^{n}$ is an interpolating sequence for $D^{\prime}$ and $Q$ is the subset of $\{1,2, \ldots, n\}$ such that $i \in Q$ iff $x_{i-1} \notin D$ and $x_{i} \notin D$, then

(1) $\Sigma_{i \in Q}\left[\left|A\left(x_{i-1}, x_{i-1}^{+}\right)\right|+\left|A\left(x_{i}^{-}, x_{i}\right)\right|\right]<\epsilon / 10$,

(2) $\Sigma_{1}^{n}\left|A^{+}\left(x_{i-1}, x_{i}\right)+A^{-}\left(x_{i-1}, x_{i}\right)-A\left(x_{i-1}, x_{i}\right)\right|<\epsilon / 10$,

$$
\begin{aligned}
\sum_{i \in Q}\left|A\left(t_{i}, x_{i}\right)\right| \leqslant \sum_{i \in Q}\left[\mid A^{+}\left(t_{i}, x_{i}\right)\right. & +A^{-}\left(t_{i}, x_{i}\right)-A\left(t_{i}, x_{i}\right) \mid \\
& \left.+\left|A^{+}\left(t_{i}, x_{i}\right)+A^{-}\left(t_{i}, x_{i}\right)\right|\right]<\epsilon / 10,
\end{aligned}
$$


and

(4) $\Sigma_{i \in Q}\left|\left[f\left(x_{i}\right)-f\left(t_{i}\right)\right]\left[g\left(t_{i}\right)-g\left(x_{i-1}\right)\right]\right|<\epsilon / 10$.

Such a subdivision $D$ exists because (1) $\left|A\left(x, x^{+}\right)\right|+\left|A\left(x^{-}, x\right)\right|=0$ except for a countable subset of $[a, b]$, (2), (3) $A^{+}+A^{-}-A \in O A^{\circ}$, and (4) Lemma 3 applies. Since $A\left(x, x^{+}\right)$and $A\left(y^{-}, y\right)$ exist and

and

$$
\lim _{p, t \rightarrow x^{+}}[f(p)-f(t)][g(t)-g(x)]=0
$$

$$
\lim _{p, t \rightarrow y^{-}}[f(p)-f(t)][g(t)-g(y)]=0
$$

for $a \leqslant x<y \leqslant b$, then there exist interpolating sequences $\left\{p_{i}\right\}_{1}^{m}$ and $\left\{q_{i}\right\}_{1}^{m}$ for $D$ such that $z_{i-1}<p_{i}<q_{i}<z_{i}$ for each $i$ and

(a) if $0<i \leqslant m$ and $p$ and $q \in\left(z_{i-1}, p_{i}\right]$, then

(5) $\left|A\left(z_{i-1}, z_{i-1}^{+}\right)-A\left(z_{i-1}, q\right)\right|<\epsilon / 10 m$; and

(6) $\left|[f(p)-f(q)]\left[g(q)-g\left(z_{i-1}\right)\right]\right|<\epsilon / 10 m$;

(b) if $0<i \leqslant m$ and $p$ and $q \in\left[q_{i}, z_{i}\right)$, then

(7) $\left|A\left(z_{i}^{-}, z_{i}\right)-A\left(p, z_{i}\right)\right|<\epsilon / 10 m$; and

(8) $\left|[f(p)-f(q)]\left[g(p)-g\left(z_{i}\right)\right]\right|<\epsilon / 10 m$.

Let $D^{\prime}=\left\{x_{i}\right\}_{0}^{n}$ be a refinement of $D \cup\left\{p_{i}\right\}_{1}^{m} \cup\left\{q_{i}\right\}_{1}^{m}$, and let $\left\{t_{i}\right\}_{1}^{n}$ be an interpolating sequence for $D^{\prime}$. Let $N, P$ and $Q$ denote subsets of $\{1,2, \ldots, n\}$ such that $i \in N$ iff $x_{i} \in D, i \in P$ iff $x_{i-1} \in D$, and $i \in Q$ iff $i \notin N \cup P$. Note that $N \cap P$ is an empty set. Since

$$
\text { (L) } \begin{aligned}
\int_{a}^{b} & \left(f d g+H+A^{+}\right)-(I) \int_{a}^{b}(f d g+H) \\
& \sim\left[f\left(x_{i-1}\right) \Delta g_{i}+H_{i}+A_{i}^{+}\right]-\left[f\left(t_{i}\right) \Delta g_{i}+H_{i}\right] \\
& =\left[f\left(x_{i-1}\right)-f\left(t_{i}\right)\right] \Delta g_{i}+A\left(x_{i-1}, x_{i-1}^{+}\right)=K_{i},
\end{aligned}
$$

then

$$
\begin{aligned}
\sum_{i \in N} K_{i}= & \sum_{i \in N}\left[f\left(x_{i-1}\right)-f\left(t_{i}\right)\right]\left[g\left(x_{i}\right)-g\left(t_{i}\right)\right] \\
& +\sum_{i \in N} A\left(x_{i-1}, x_{i-1}^{+}\right)-\sum_{i \in N} A\left(x_{i-1}, t_{i}\right),
\end{aligned}
$$

and it follows from inequalities 8,1 and 3 that $\Sigma_{i \in N}\left|K_{i}\right|<\epsilon / 10+\epsilon / 10+\epsilon / 10$; also

$$
\begin{aligned}
\sum_{i \in P} K_{i}= & \sum_{i \in P}\left[f\left(x_{i}\right)-f\left(t_{i}\right)\right]\left[g\left(x_{i}\right)-g\left(x_{i-1}\right)\right] \\
& +\sum_{i \in P}\left[A\left(x_{i-1}, x_{i-1}^{+}\right)-A\left(x_{i-1}, x_{i}\right)\right],
\end{aligned}
$$

and from inequalities 6 and $5, \Sigma_{i \in P}\left|K_{i}\right|<\epsilon / 10+\epsilon / 10$; also, 


$$
\begin{aligned}
\sum_{i \in Q} K_{i}= & \sum_{i \in Q}\left[f\left(x_{i}\right)-f\left(t_{i}\right)\right]\left[g\left(x_{i}\right)-g\left(t_{i}\right)\right] \\
& +\sum_{i \in Q}\left[f\left(x_{i}\right)-f\left(t_{i}\right)\right]\left[g\left(t_{i}\right)-g\left(x_{i-1}\right)\right] \\
& +\sum_{i \in Q}\left(A_{i}^{+}+A_{i}^{-}-A_{i}\right)-\sum_{i \in Q} A_{i}^{-}
\end{aligned}
$$

and from inequalities $3,4,2$ and 1 ,

$$
\sum_{i \in Q}\left|K_{i}\right|<\epsilon / 10+\epsilon / 10+\epsilon / 10+\epsilon / 10 .
$$

Hence, $\Sigma_{1}^{n}\left|K_{i}\right|<\epsilon$. Therefore, if either of the following integrals exists, then $(L) \int_{a}^{b}\left(f d g+H+A^{+}\right)=(I) \int_{a}^{b}(f d g+H)$.

Since $0=\int_{a}^{b}\left(A-A^{+}-A^{-}\right)=(R L) \int_{a}^{b}\left[(f d g-f d g)-A^{+}-A^{-}\right]$, it follows that if either of the following integrals exists, then

$$
\begin{aligned}
(L) \int_{a}^{b}\left(f d g+H+A^{+}\right) \\
\quad=(L) \int_{a}^{b}\left(f d g+H+A^{+}\right)+(R L) \int_{a}^{b}\left[(f d g-f d g)-A^{+}-A^{-}\right] \\
\quad=(R) \int_{a}^{b}\left(f d g+H-A^{-}\right) .
\end{aligned}
$$

THEOREM 2. Given. $f$ and $g$ are bounded functions from $R$ to $R$, and $H$ is a function from $R \times R$ to $R$.

Conclusion. If $d g \in O B^{\circ}$ on $[a, b]$, then Statements 1,2 and 3 are equivalent and the integrals are equal on each subinterval of $[a, b]$. If $d f \in O B^{\circ}$ and $A^{+}$and $A^{-}$exist on $[a, b]$, then Statements 1 and 2 are equivalent and the integrals are equal on each subinterval of $[a, b]$.

1. (I) $\int_{a}^{b}(f d g+H)$ exists.

2. If $a \leqslant x<y \leqslant b$, then $A\left(x, x^{+}\right)$and $A\left(y^{-}, y\right)$ exist, the following integrals exist, and

$$
\text { (L) } \int_{x}^{y}\left(f d g+H+A^{+}\right)=(R) \int_{x}^{y}\left(f d g+H-A^{-}\right) .
$$

3. If $a \leqslant x<y \leqslant b$, then $A\left(x, x^{+}\right)$and $A\left(y^{-}, y\right)$ exist, the following integrals exist, and

$$
(L R) \int_{x}^{y}\left[f\left(d g-G^{+}\right)+f G^{+}+H\right]=(L R) \int_{x}^{y}\left[f G^{-}+f\left(d g-G^{-}\right)+H\right],
$$

where $G(r, t)=g(t)-g(r), G^{+}(r, t)=G\left(r, r^{+}\right)$, and $G^{-}(r, t)=G\left(t^{-}, t\right)$.

PROOF. If $(I) \int_{a}^{b}(f d g+H)$ exists and either $d f$ or $d g \in O B^{\circ}$ on $[a, b]$, then 


$$
\begin{aligned}
0 & =\lim _{y, p, q \rightarrow x^{+}}\{[f(p)-f(q)][g(y)-g(x)]+[f(p)-f(q)][g(q)-g(y)]\} \\
& =\lim _{p, q \rightarrow x^{+}}[f(p)-f(q)][g(q)-g(x)]
\end{aligned}
$$

where $p, q \in(x, y)$. The limit of the first term is zero because $(I) \int_{a}^{b}(f d g+H)$ exists; the limit of the second term is zero because $f$ and $g$ are bounded and either $d g \in O B^{\circ}$ or $d f \in O B^{\circ}$. Similarly, if $x \in(a, b]$, then

$$
\lim _{p, q \rightarrow x^{-}}[f(p)-f(q)][g(q)-g(x)]=0 .
$$

Proof for $1 \rightarrow 2$, where $A^{+}$and $A^{-}$exist and $d f \in O B^{\circ}$ on $[a, b]$. We will show that $\int_{p}^{q}\left(A-A^{+}-A^{-}\right)=0$ for $a \leqslant p<q \leqslant b$; then Conclusion 2 follows from Theorem 1. Suppose that $\epsilon>0$ and $a \leqslant p<q \leqslant b$. Since $d f$ and $A \in O B^{\circ}$ on $[p, q]$, there is a finite subset $J$ of $[p, q]$ such that on $[p, q]$

$$
\sum_{x \notin J}\left[\left|A\left(x, x^{+}\right)\right|+\left|A\left(x^{-}, x\right)\right|\right]<\epsilon / 10,
$$

and

$$
\sum_{x \notin J}\left[\left|f(x)-f\left(x^{-}\right)\right|+\left|f(x)-f\left(x^{+}\right)\right|\right]<\epsilon / 20 m,
$$

where $m$ is a bound for $|g|$. Since $(I) \int_{p}^{q}(f d g+H)$ exists, then the multivalued function $f d g+H$ has the $O A^{\circ}$ property; hence, there is a subdivision $D_{1}$ of $[p, q]$ such that, if $D^{\prime}=\left\{x_{i}\right\}_{0}^{n}$ is a refinement of $D_{1}$ and $\left\{r_{i}\right\}_{1}^{n}$ and $\left\{t_{i}\right\}_{1}^{n}$ are interpolating sequences for $D^{\prime}$, then

$$
\sum_{1}^{n}\left|\left[f\left(t_{i}\right)-f\left(r_{i}\right)\right]\left[g\left(x_{i}\right)-g\left(x_{i-1}\right)\right]\right|<\epsilon / 20 .
$$

Since $d f \in O B^{\circ}$ on $[p, q]$, there is a subdivision $D$ of $[p, q]$ which is a refinement of $D_{1} \cup J$ such that a subset of $D$ is an interpolation sequence for $D_{1} \cup J$ and, if $\left\{x_{i}\right\}_{0}^{n}$ is a refinement of $D$ and $N, P$ and $Q$ are subsets of $\{1,2$, $\ldots, n\}$ such that $i \in N$ iff $x_{i} \in D_{1} \cup J, i \in P$ iff $x_{i-1} \in D_{1} \cup J$, and $i \in Q$ iff $i \notin N \cup P$, then

$$
\sum_{i \in N}\left|A\left(x_{i-1}, x_{i}\right)-A\left(x_{i}^{-}, x_{i}\right)\right|+\sum_{i \in P}\left|A\left(x_{i-1}, x_{i}\right)-A\left(x_{i-1}, x_{i-1}^{+}\right)\right|<\epsilon / 10,
$$

and

$$
\sum_{i \in Q}\left[\left|f\left(x_{i}\right)-f\left(x_{i}^{-}\right)\right|+\left|f\left(x_{i-1}^{+}\right)-f\left(x_{i-1}\right)\right|\right]\left|\Delta g_{i}\right|<\epsilon / 10 \text {. }
$$

Therefore, if $D^{\prime}=\left\{x_{i}\right\}_{0}^{n}$ is a refinement of $D$ and $N, P$ and $Q$ are sets of integers as defined above, then 


$$
\begin{aligned}
& \sum_{1}^{n}\left|A\left(x_{i-1}, x_{i}\right)-A^{+}\left(x_{i-1}, x_{i}\right)-A^{-}\left(x_{i-1}, x_{i}\right)\right| \\
& \leqslant \sum_{i \in N}\left|A\left(x_{i-1}, x_{i}\right)-A\left(x_{i}^{-}, x_{i}\right)\right|+\sum_{i \in N}\left|A\left(x_{i-1}, x_{i-1}^{+}\right)\right| \\
& \quad+\sum_{i \in P}\left|A\left(x_{i-1}, x_{i}\right)-A\left(x_{i-1}, x_{i-1}^{+}\right)\right|+\sum_{i \in P}\left|A\left(x_{i}^{-}, x_{i}\right)\right| \\
& \quad+\sum_{i \in Q}\left|f\left(x_{i}\right)-f\left(x_{i}^{-}\right)\right|\left|\Delta g_{i}\right|+\sum_{i \in Q}\left|f\left(x_{i}^{-}\right)-f\left(x_{i-1}^{+}\right)\right|\left|\Delta g_{i}\right| \\
& \quad+\sum_{i \in Q}\left|f\left(x_{i-1}^{+}\right)-f\left(x_{i-1}\right)\right|\left|\Delta g_{i}\right| \\
& \quad+\sum_{i \in Q}\left[\left|A\left(x_{i-1}, x_{i-1}^{+}\right)\right|+\left|A\left(x_{i}^{-}, x_{i}\right)\right|\right]<\epsilon .
\end{aligned}
$$

(By using inequalities 4, 1, 4, 1, 2, 3, 2 and 1 in this order, it can be shown that each summation above is less than $\epsilon / 10$.) Therefore, $\int_{p}^{q}\left(A-A^{+}-A^{-}\right)$exists and is zero. Conclusion 2 for $d f \in O B^{\circ}$ follows from Theorem 1 .

Proof for $1 \rightarrow 2$, where $d g \in O B^{\circ}$. Suppose $x \in[a, b)$. If $g\left(x^{+}\right)=g(x)$, then $A^{+}(x, p)=\lim _{p \rightarrow x^{+}}[f(p)-f(x)][g(p)-g(x)]=0$. Suppose that $g\left(x^{+}\right) \neq$ $g(x)$. Since $\lim _{p, q \rightarrow x^{+}}[f(p)-f(q)][g(p)-g(x)]=0$, then $f\left(x^{+}\right)$exists and $A^{+}(x, p)=\left[f\left(x^{+}\right)-f(x)\right]\left[g\left(x^{+}\right)-g(x)\right]$. Therefore, $A^{+}$exists on $[a, b]$. Similarly, $A^{-}$exists on $[a, b]$.

The proof that $\int_{p}^{q}\left(A-A^{+}-A^{\top}\right)$ exists and is zero is similar to the proof where $d f \in O B^{\circ}$ except for showing that $\Sigma_{i \in Q}\left|A_{i}\right|<\epsilon / 4$. Note that,

$$
\sum_{i \in Q}\left|A_{i}\right|=\sum_{i \in Q}\left|\left(f_{i}-f_{i-1}\right)\left(g_{i}-g_{i-1}\right)\right| \leqslant \alpha+\beta,
$$

where

$$
\alpha=\sum_{i \in Q}\left[\left|\Delta f_{i}\right|\left|g_{i}-g_{i}^{+}\right|+\left|\Delta f_{i}\right|\left|g_{i-1}^{-}-g_{i-1}\right|\right]
$$

and $\beta=\Sigma_{i \in Q}\left|\Delta f_{i}\right|\left|g_{i}^{+}-g_{i-1}^{-}\right|$. Since $d g \in O B^{\circ}$ and $f$ is bounded, subdivisions can be defined so that $\alpha<\epsilon / 10$. Since $(I) \int_{a}^{b}(f d g+H)$ exists, subdivisions can be defined so that $\beta<\epsilon / 10$. Hence, $\Sigma_{i \notin Q}\left|A_{i}\right|+\Sigma_{i \in Q}\left|A_{i}\right|$ can be made arbitrarily small. Therefore, $\int_{p}^{q}\left(A-A^{+}-A^{-}\right)$exists and is zero, and Conclusion 2 for $d g \in O B^{\circ}$ follows from Theorem 1 .

Proof of $2 \rightarrow 1$, where $d g \in O B^{\circ}$. It follows from Conclusion 2 that

$$
0=(R L) \int_{x}^{y}\left[(f d g-f d g)-A^{+}-A^{-}\right]=\int_{x}^{y}\left(A-A^{+}-A^{-}\right)
$$

for $a \leqslant x<y \leqslant b$. Suppose $x \in[a, b)$. Since $A^{+}$exists, then

$$
\lim _{y \rightarrow x^{+}}[f(y)-f(x)][g(y)-g(x)]
$$

exists. If $\lim _{y \rightarrow x^{+}}[g(y)-g(x)] \neq 0$, then $\lim _{y \rightarrow x^{+}}[f(y)-f(x)]$ exists and $\lim _{p, q \rightarrow x^{+}}[f(p)-f(q)]=0$. Since $f$ and $g$ are bounded functions and since one 
of $\lim _{p, q \rightarrow x^{+}}[f(p)-f(q)]$ or $\lim _{q \rightarrow x^{+}}[g(q)-g(x)]$ is zero, then

$$
\lim _{p, q \rightarrow x^{+}}[f(p)-f(q)][g(q)-g(x)]=0 .
$$

Similarly,

$$
\lim _{p, q \rightarrow x^{-}}[f(p)-f(q)][g(q)-g(x)] \cdot=0 .
$$

It follows from Theorem 1 that $(I) \int_{x}^{y}(f d g+H)$ exists and is $(L) \int_{x}^{y}\left(f d g+H+A^{+}\right)$.

Proof of $2 \rightarrow 1$, where $d f \in O B^{\circ}$. Since $d f \in O B^{\circ}$, then

$$
\lim _{p, q \rightarrow x^{+}}[f(p)-f(q)]=0 \text { and } \lim _{p, q \rightarrow y^{-}}[f(p)-f(q)]=0
$$

for $a \leqslant x<y \leqslant b$; therefore, equations (5) and (6) hold. Since $A^{+}$and $A^{-}$exist and $\int_{x}^{y}\left(A-A^{+}-A^{-}\right)=0$ for $a \leqslant x<y \leqslant b$, it follows from Theorem 1 that (I) $\int_{x}^{y}(f d g+H)$ exists and is $(L) \int_{x}^{y}\left(f d g+H+A^{+}\right)$.

We will now prove that the following integrals exist and that $\int_{x}^{y} A^{+}=$ $(R L) \int_{x}^{y}\left(f G^{+}-f G^{+}\right)$and $\int_{x}^{y} A^{-}=(R L) \int_{x}^{y}\left(f G^{-}-f G^{-}\right)$, where $d g \in O B^{\circ}, A^{+}$ and $A^{-}$exist, and $G, G^{+}$and $G^{-}$are defined as in Conclusion 3. Then these results will be used to prove $2 \leftrightarrow 3$. Since $A^{+}$and $A^{-}$exist and $A \in O B^{\circ}$, then $\int_{a}^{b} A^{+}$and $\int_{a}^{b} A^{-}$exist.

Suppose that $a \leqslant x<y \leqslant b, \epsilon>0$, and $M$ is an upper bound for $|f|$ on $[a, b]$. Since $d g \in O B^{\circ}$ and since $A^{+}$exists on $[a, b]$, there is a finite subset $J$ of $[x, y]$ such that $(2 M+1) \Sigma_{x \notin J}\left|G\left(x, x^{+}\right)\right|<\epsilon / 2$ and, if $x \in J$, then $G\left(x, x^{+}\right)$ $\neq 0$; hence, if $x \in J$, then $f\left(x^{+}\right)$exists. Let $K=J \cup\{a, b\}=\left\{z_{i}\right\}_{0}^{m}$; then there is an interpolating sequence $\left\{t_{i}\right\}_{1}^{m}$ for $K$ such that if $z_{i-1} \in J$ and $z_{i-1}<p<t_{i}$, then $\left|f\left(z_{i-1}^{+}\right)-f(p)\right|<\epsilon / 2 V$, where $V=\Sigma_{x \in[a, b)}\left|G\left(x, x^{+}\right)\right|$.

Let $D=J \cup\{a, b\} \cup\left\{t_{i}\right\}_{1}^{m}$, and let $D^{\prime}=\left\{x_{i}\right\}_{0}^{n}$ be a refinement of $D$. Let $f\left(x^{*}\right)$ denote a cluster value of $f$ at $x^{+}$; then $A^{+}(x, y)=\left[f\left(x^{*}\right)-f(x)\right] G^{+}(x, y)$, and

$$
\begin{aligned}
\sum_{1}^{n}\left|A^{+}\left(x_{i-1}, x_{i}\right)-\left[f\left(x_{i}\right)-f\left(x_{i-1}\right)\right] G^{+}\left(x_{i-1}, x_{i}\right)\right| \\
\quad=\sum_{1}^{n}\left|\left[f\left(x_{i-1}^{*}\right)-f\left(x_{i-1}\right)\right] G_{i}^{+}-\left[f\left(x_{i}\right)-f\left(x_{i-1}\right)\right] G_{i}^{+}\right| \\
\quad=\sum_{1}^{n}\left|\left[f\left(x_{i-1}^{*}\right)-f\left(x_{i}\right)\right] G_{i}^{+}\right| \\
\quad \leqslant \sum_{x_{i-1} \in J}\left|\left[f\left(x_{i-1}^{+}\right)-f\left(x_{i}\right)\right] G_{i}^{+}\right|+\sum_{x_{i-1} \notin J}\left|\left[f\left(x_{i-1}^{*}\right)-f\left(x_{i}\right)\right] G_{i}^{+}\right| \\
\quad<(\epsilon / 2 V) \sum_{x_{i-1} \in J}\left|G\left(x_{i-1}, x_{i-1}^{+}\right)\right|+\epsilon / 2<\epsilon .
\end{aligned}
$$


Hence, $\int_{x}^{y} A^{+}=(R L) \int_{x}^{y}\left(f G^{+}-f G^{+}\right)$and, similarly, $\int_{x}^{y} A^{-}=(R L) \int_{x}^{y}\left(f G^{-}-f G^{-}\right)$.

Proof of $2 \leftrightarrow 3$, where $d g \in O B^{\circ}$. If Conclusion 2 holds, then the following integrals exist and $\int_{x}^{y} A^{+}=(R L) \int_{x}^{y}\left(f G^{+}-f G^{+}\right)$and

$$
\int_{x}^{y} A^{-}=(R L) \int_{x}^{y}\left(f G^{-}-f G^{-}\right)
$$

By combining the above equations with the equations in Conclusion 2, one can show that

$$
(L) \int_{x}^{y}\left(f d g+H+A^{+}\right)=(L R) \int_{x}^{y}\left[f\left(d g-G^{+}\right)+f G^{+}+H\right]
$$

and

$$
(R) \int_{x}^{y}\left(f d g+H-A^{-}\right)=(L R) \int_{x}^{y}\left[f G^{-}+f\left(d g-G^{-}\right)+H\right] ;
$$

hence, $2 \rightarrow 3$.

The proof for $3 \rightarrow 2$, with $d g \in O B^{\circ}$, is identical to the above proof of $2 \rightarrow 3$.

The following example shows that $d f \in O B^{\circ}$ and the existence of $(I) \int_{a}^{b} f d g$ does not imply that $A^{+}$and $A^{-}$exist. Let $g$ be a function such that $g\left(b^{-}\right)$does not exist, and let $f$ be the function such that $f(b)=2$ and $f(x)=1$ for $x \in$ $[a, b)$. Then $(I) \int_{a}^{b} f d g=g(b)-g(a)$, but $A\left(b^{-}, b\right)$ does not exist.

It is possible for the function $A(x, y)=[f(y)-f(x)][g(y)-g(x)]$ to belong to $O A^{\circ}$ and $O B^{\circ}$ and neither $f$ nor $g$ to have bounded variation

EXAMPLE. Let $f(x)=\sin (1 / x)$ for $x<0$ and $f(x)=0$ for $x \geqslant 0$, and $g(x)=0$ for $x \leqslant 0$ and $g(x)=\sin (1 / x)$ for $x>0$; on the interval $[-1,1]$, $A \in O A^{\circ}$ and $O B^{\circ}$ and $\int_{p}^{q} A=0$ for $-1 \leqslant p<q \leqslant 1$; however, $d f \notin O B^{\circ}$ and $d g \notin O B^{\circ}$.

Theorem 5.1 of [3] gives a set of necessary and sufficient conditions for the integral equation $f(x)=h(x)+(L R) \int_{a}^{x}(f U+f V)$ to have a solution, where $U$ and $V$ are functions from $R \times R$ to $R$. We now show that Theorem 5.1 of [3], used with Theorem 2, gives a method for finding the solution of the equation $f(x)=h(x)+(I) \int_{a}^{x}(f d g+H)$, where $H(x, y)=f(x) K(x, y)+f(y) F(x, y)+$ $Q(x, y)$ and $Q$ is independent of $f$ and $\int_{a}^{x} Q$ exists. Suppose that $G$ and $G^{+}$are defined as in Conclusion 3 of Theorem 2, and that suitable restrictions are placed on each function; then,

$$
\begin{aligned}
f(x) & =h(x)+(I) \int_{a}^{x}(f d g+H) \\
& =h(x)+(L R) \int_{a}^{x}\left[f\left(d g-G^{+}\right)+f G^{+}+H\right] \quad(\text { Theorem 2) } \\
& =h(x)+(L R L R) \int_{a}^{x}\left[f\left(d g-G^{+}\right)+f G^{+}+f K+f F+Q\right]
\end{aligned}
$$

and 


$$
f(x)=\left[h(x)+\int_{a}^{x} Q\right]+(L R) \int_{a}^{x}\left[f\left(d g-G^{+}+K\right)+f\left(G^{+}+F\right)\right]
$$

Theorem 5.1 of [3] gives the solution for the preceding equation.

It is easily proved that, if the function $d g$ in Theorem 2 is replaced by a function $G$ from $R \times R$ to $R$ such that $G \in O A^{\circ}$ and $O B^{\circ}$ on $[a, b]$, then the resulting Conclusions 1,2 , and 3 are equivalent. The proof of the following theorem (which is used in the proof of Theorem 5) illustrates a method for constructing such proofs.

THEOREM 3. Given. $f$ is a bounded function from $R$ to $R$, and $Q, Q^{+}$and $H$ are functions from $R \times R$ to $R$ such that $Q \in O A^{\circ}$ and $O B^{\circ}$ on $[a, b]$, $(I) \int_{a}^{b}(f Q+H)$ exists, and $Q^{+}(x, y)=Q\left(x, x^{+}\right)$for $x \in[a, b)$, provided $Q\left(x, x^{+}\right)$ exists.

Conclusion. If $a \leqslant x<y \leqslant b$, then $Q^{+}(x, y)$ exists, and

$$
(L R) \int_{x}^{y}\left[f\left(Q-Q^{+}\right)+f Q^{+}+H\right]
$$

exists and is $(I) \int_{x}^{y}(f Q+H)$.

Proof. Since $Q \in O A^{\circ}$ and $O B^{\circ}$, then $Q\left(x, x^{+}\right)$exists for $x \in[a, b)$. Let $g$ and $G$ be functions such that $g(x)=\int_{a}^{x} Q$ and $G(x, y)=\int_{x}^{y} Q$ for $a \leqslant x<y \leqslant b$. Since $d g \in O B^{\circ}$, then the triple $f, g, H$ satisfies Conclusion 1 of Theorem 2; hence Conclusion 3 holds and

$$
\text { (I) } \int_{x}^{y}(f d g+H)=(L R) \int_{x}^{y}\left[f\left(d g-G^{+}\right)+f G^{+}+H\right] .
$$

Since $Q \in O A^{\circ}$, then $0=\int_{x}^{y}\left(Q-\int Q\right)=\int_{x}^{y}(Q-G)=\int_{x}^{y}(Q-d g)$ and $G\left(x, x^{+}\right)=$ $Q\left(x, x^{+}\right)=Q^{+}(x, y)$ for $a \leqslant x<y \leqslant b$. Therefore,

and

$$
(I) \int_{x}^{y} f(d g-Q)=(I) \int_{x}^{y} f(G-Q)=(I) \int_{x}^{y} f\left(\int Q-Q\right)=0
$$

$$
\begin{aligned}
& (I) \int_{x}^{y}(f Q+H)=(I) \int_{x}^{y}[f(Q+d g-Q)+H]=(I) \int_{x}^{y}(f d g+H) \\
& \quad=(L R) \int_{x}^{y}\left[f\left(d g-G^{+}\right)+f G^{+}+H\right]=(L R) \int_{x}^{y}\left[f\left(Q-Q^{+}\right)+f Q^{+}+H\right]
\end{aligned}
$$

for $a \leqslant x<y \leqslant b$.

THEOREM 4. Given. $[a, b]$ is a number interval, $f$ and $g$ are bounded functions from $R$ to $R$, and $H$ is a function from $R \times R$ to $R$.

Conclusion. 1. If $A^{+}$and $A^{-}$exist on $[a, b]$, if $d g$ or $d f \in O B^{\circ}$ on $[a, b]$, and if $(I) \int_{a}^{b}(H+f d g)$ or $(I) \int_{a}^{b}(H-g d f)$ exists, then the other integral exists and

$$
(I) \int_{a}^{b}(H+f d g)=(I) \int_{a}^{b}(H-g d f)+f(b) g(b)-f(a) g(a)+\int_{a}^{b}\left(A^{+}-A^{-}\right) .
$$


2. If $d g \in O B^{\circ}$ on $[a, b]$ and $(I) \int_{a}^{b}(H+f d g)$ exists, then $A^{+}$and $A^{-}$exist on $[a, b]$, and the equation in Conclusion 1 holds.

Proof. Suppose $A^{+}$and $A^{-}$exist, and suppose $(I) \int_{a}^{b}(H+f d g)$ exists. If either $d f \in O B^{\circ}$ or $d g \in O B^{\circ}$, then $\int_{a}^{b} A^{+}$and $\int_{a}^{b} A^{-}$exist, and

$$
\begin{aligned}
& (I) \int_{a}^{b}(f d g+H)=(L) \int_{a}^{b}\left(f d g+H+A^{+}\right) \quad(\text { Theorem } 2,1 \rightarrow 2) \\
& \quad=(R) \int_{a}^{b}\left(-g d f+H+A^{+}\right)+f(b) g(b)-f(a) g(a) \\
& \quad=-(R) \int_{a}^{b}\left(g d f-H-A^{-}\right)+f(b) g(b)-f(a) g(a)+\int_{a}^{b}\left(A^{+}-A^{-}\right) \\
& \left.\quad=-(I) \int_{a}^{b}(g d f-H)+f(b) g(b)-f(a) g(a)+\int_{a}^{b}\left(A^{+}-A^{-}\right) \quad \text { (Theorem 2,2 } \rightarrow 1\right) .
\end{aligned}
$$

If $(I) \int_{a}^{b}(H-g d f)$ exists, similar manipulations will hold.

If $d g \in O B^{\circ}$ and $(I) \int_{a}^{b}(H+f d g)$ exists, it follows from Theorem 2, $1 \rightarrow 2$, that $A^{+}$and $A^{-}$exist; hence, the preceding manipulations hold for this case also.

If $f$ and $g$ satisfy the hypothesis of Theorem 4 , then the following relationships can be proved as corollaries to Theorem 4 .

$$
\begin{aligned}
& (L I R) \int_{a}^{b}(u d r+f d g+v d s) \\
& =f(b) g(b)-f(a) g(a)+(L I R) \int_{a}^{b}(u d r-g d f+v d s)+\int_{a}^{b}\left(A^{+}-A\right) \\
& =f(b) g(b)-f(a) g(a)+u(b) r(b)-u(a) r(a) \\
& \quad+(R I R) \int_{a}^{b}(-r d u-g d f+v d s)+\int_{a}^{b}\left(A^{+}-A^{-}\right) \\
& =f(b) g(b)-f(a) g(a)+u(b) r(b)-u(a) r(a)+v(b) s(b) \\
& \quad-v(a) s(a)+(R I L) \int_{a}^{b}(-r d u-g d f-s d v)+\int_{a}^{b}\left(A^{+}-A^{-}\right) .
\end{aligned}
$$

In a recent paper Wright and Baker prove an integration-by-parts theorem [13, Theorem 3.2] for the integral $(L I R) \int_{a}^{b}\left(w_{1} f d g+w_{2} f d g+w_{3} f d g\right)$. Their Theorem 3.2 can be proved as a corollary to Theorem 4 above in which $w_{2} f(t)$ and $\left[w_{1} f(x)+w_{3} f(y)\right][g(y)-g(x)]$ of Theorem 3.2 play the roles of $f(t)$ and $H(x, y)$, respectively, in Theorem 4.

THEOREM 5. Given. $[a, b]$ is a number interval, $m$ and $c$ are numbers, and $h$ and $k$ are bounded functions from $R$ to $R$ such that $k \geqslant 0$ and $d h \in O B^{\circ}$ on $[a, b] ; H, G$ and $K$ are functions from $R \times R$ to $R$ which belong to $O A^{\circ}$ and $O B^{\circ}$ on $[a, b] ; S$ is the set of functions such that $f \in S$ iff $f$ is a bounded nonnegative function from $R$ to $R$ such that if $x \in[a, b]$ then the following integral exists and

$$
f(x) \leqslant h(x)+(L I R) \int_{a}^{x}(f k H+f k G+f k K)
$$


$u, v, U, V$ and $G^{+}$are functions from $R \times R$ to $R$ such that $U=(1 / 2)(|u|+u)$, $V=(1 / 2)(|v|+v)$ and $G^{+}(x, y)=G\left(x, x^{+}\right)$.

Conclusion 1. If $m=\operatorname{lub}_{x \in[a, b]} k(x), 1-m\left(G^{+}+K\right) \geqslant c>0$ on $[a, b]$, and $u(x, y)=H(x, y)+G(x, y)-G\left(x, x^{+}\right)$and $v(x, y)=G\left(x, x^{+}\right)+K(x, y)$ for $a \leqslant x<y \leqslant b$, then the function $g$ such that

$$
\begin{aligned}
g(x)= & h(a)_{a} \Pi^{x}(1+m U)(1-m V)^{-1} \\
& +(R) \int_{a}^{x}(1-m V)^{-1}{ }_{t} \Pi^{x}(1+m U)(1-m V)^{-1} d h
\end{aligned}
$$

exists on $[a, b]$; furthermore, if $f \in S$ and $x \in[a, b]$, then $f(x) \leqslant g(x)$.

Conclusion 2. If $k$ is quasi-continuous, and $u, v, U$ and $V$ are defined as in Conclusion 1 , and $|k U|(x, y)=|k(x) U(x, y)|$ and $|k V|(x, y)=|k(y) V(x, y)|$, and $1-|k V| \geqslant c>0$, then the function $g$ such that

$$
\begin{aligned}
g(x)= & h(a)_{a} \Pi^{x}(1+|k U|)(1-|k V|)^{-1} \\
& +(R) \int_{a}^{x}(1-|k V|)^{-1}{ }_{t} \Pi^{x}(1+|k U|)(1-|k V|)^{-1} d h
\end{aligned}
$$

exists on $[a, b]$. Furthermore, if $f \in S$ and $x \in[a, b]$, then $f(x) \leqslant g(x)$; also, if $u(x, y) \geqslant 0$ and $v(x, y) \geqslant 0$ on $[a, b]$, then $g \in S$.

Conclusion 3. If $k$ is quasi-continuous and $P, Q, T, u$ and $v$ are functions such that $P(x, y)=k(x) H(x, y), Q(x, y)=k\left(x^{+}\right) G(x, y), T(x, y)=k(y) K(x, y)$, $u(x, y)=P(x, y)+Q(x, y)-Q\left(x, x^{+}\right)$and $v(x, y)=Q\left(x, x^{+}\right)+T(x, y)$ for $a \leqslant x<y \leqslant b$ and $1-\left(Q^{+}+T\right) \geqslant c>0$ on $[a, b]$, then the function $g$ such that

$$
g(x)=h(a)_{a} \Pi^{x}(1+U)(1-V)^{-1}+(R) \int_{a}^{x}(1-V)^{-1}{ }_{t} \Pi^{x}(1+U)(1-V)^{-1} d h
$$

exists on $[a, b]$; furthermore, $g \in S$ and, if $f \in S$ and $x \in[a, b]$, then $f(x) \leqslant g(x)$. then

Proof of Conclusion 1. Let $g$ be the function such that, if $x \in[a, b]$,

$$
\begin{aligned}
g(x)= & h(a)_{a} \Pi^{x}(1+m U)(1-m V)^{-1} \\
& +(R) \int_{a}^{x}(1-m V)^{-1}\left[\Pi_{t}^{x}(1+m U)(1-m V)^{-1}\right] d h .
\end{aligned}
$$

Since $u$ and $v \in O A^{\circ}$ and $O B^{\circ}$ on $[a, b]$, it follows from Lemma 4 that $m U$ and $m V \in O A^{\circ}$ and $O B^{\circ}$. Since $1-m V \geqslant c>0$, it follows from Lemma 5 that $g$ exists on $[a, b]$ and that, if $x \in[a, b]$, then $g(x)=h(x)+(L R) \int_{a}^{x}(g m U+g m V)$.

Suppose that $f \in S$ and $x \in(a, b]$. Since $G \in O B^{\circ}$ and $O A^{\circ}$, it follows from Theorem 3, with $f k$ playing the role of $f$, that

$$
(L I R) \int_{a}^{x}(f k H+f k G+f k K)=(L R) \int_{a}^{x}[(f k) u+(f k) v]
$$

and, hence, $f(x) \leqslant h(x)+(L R) \int_{a}^{x}[(f k) u+(f k) v]$. If $\epsilon>0$, there is a subdivi- 
sion $D$ of $[a, x]$ such that if $\left\{x_{i}\right\}_{0}^{n}$ is a refinement of $D$ then there is a number $e$ such that $|e|<\epsilon$ and such that

$$
\begin{array}{r}
f(x)-g(x) \leqslant(L R) \int_{a}^{x}(f k u+f k v)-(L R) \int_{a}^{x}(g m U+g m V) \\
=\sum_{1}^{n}\left(f_{i-1} k_{i-1} u_{i}+f_{i} k_{i} v_{i}-g_{i-1} m U_{i}-g_{i} m V_{i}\right)+e .
\end{array}
$$

Since $f_{i-1} m U_{i} \geqslant f_{i-1} k_{i-1} u_{i}$ and $f_{i} m V_{i} \geqslant f_{i} k_{i} v_{i}$ for each $i$, then

$$
\begin{aligned}
f(x)-g(x) & \leqslant \sum_{1}^{n}\left(f_{i-1} m U_{i}+f_{i} m V_{i}-g_{i-1} m U_{i}-g_{i} m V_{i}\right)+e \\
& \leqslant \sum_{1}^{n}\left[\left(f_{i-1}-g_{i-1}\right) m U_{i}+\left(f_{i}-g_{i}\right) m V_{i}\right]+\epsilon .
\end{aligned}
$$

Since $f(a) \leqslant g(a)$ and $f-g$ is bounded on $[a, b]$ and $m U$ and $m V$ are nonnegative functions such that $m U$ and $m V \in O A^{\circ}$ and $O B^{\circ}$ and such that $1-m V \geqslant c>0$, it follows from Lemma 6 that $f(x) \leqslant g(x)$ for $x \in[a, b]$.

Proof of Conclusion 2. Suppose that $g$ is the function defined in Conclusion 2 and that $k$ is quasi-continuous on $[a, b]$. Since each of $u$ and $v \in O A^{\circ}$ and $O B^{\circ}$ and $k$ is quasi-continuous on $[a, b]$, it follows from Lemma 2 that $k u$ and $k v \in O A^{\circ}$ and $O B^{\circ}$ and from Lemmas 2 and 4 that $|k U|$ and $|k V| \in O A^{\circ}$ and $O B^{\circ}$ on $[a, b]$; also, $1-|k V| \geqslant c>0,|k U| \geqslant 0$ and $|k V| \geqslant 0$. A repetition of the steps in Conclusion 1 (with $m U$ and $m V$ replaced by $|k U|$ and $|k V|$ ) shows that $g$ exists and is $h(x)+(L R) \int_{a}^{x}(g|k U|+g|k V|)$ on $[a, b]$ and, if $f \in S$ and $x \in[a, b]$, then $f(x) \leqslant g(x)$.

If each of $u$ and $v$ is nonnegative on $[a, b]$, then $U=(1 / 2)(|u|+u)=u$ and $V=(1 / 2)(|v|+v)=v$, and $k(x) u(x, y)=|k U|(x, y)$ and $k(y) v(x, y)=$ $|k V|(x, y)$ on $[a, b]$. We now show that $g \in S$. Since $g$ and $k$ are quasi-continuous and each of $H, G$ and $K \in O A^{\circ}$ and $O B^{\circ}$, then by Lemma 2 each of $(L) \int_{a}^{b} g k H$, $(I) \int_{a}^{b} g k G$ and $(R) \int_{a}^{b} g k K$ exists; hence, $(L I R) \int_{a}^{x}(g k H+g k G+g k K)$ exists for $x \in[a, b]$ and, by Theorem 3 , is $(L R) \int_{a}^{x}(g k u+g k v)$. Hence,

$$
\begin{aligned}
g(x) & =h(x)+(L R) \int_{a}^{x}(g|k U|+g|k V|) \\
& =h(x)+(L R) \int_{a}^{x}(g k u+g k v) \\
& =h(x)+(L I R) \int_{a}^{x}(g k H+g k G+g k K) \text { (Theorem 3) }
\end{aligned}
$$

for $x \in[a, b]$. Therefore, $g \in S$.

Proof of Conclusion 3. Suppose $k$ is quasi-continuous on $[a, b]$ and that $P, Q, T, u$ and $v$ are functions with the properties given in Conclusion 3. Since $H, G$ and $K \in O A^{\circ}$ and $O B^{\circ}$ and $k$ is quasi-continuous, it follows from Lemma 2 that $P, Q$ and $T \in O A^{\circ}$ and $O B^{\circ}$ on $[a, b]$. The functions $P, Q, T, u$, 
$v, U$ and $V$ satisfy the hypothesis for Conclusion 1 (with $P, Q, T$ playing the roles of $H, G, K$, respectively), and with $k=1$; hence,

$$
f(x) \leqslant h(x)+(L I R) \int_{a}^{x}(f P+f Q+f T)
$$

and the function $g$ such that

$$
g(x)=h(a)_{a} \Pi^{x}(1+U)(1-V)^{-1}+(R) \int_{a}^{x}(1-V)^{-1} \Pi_{t}^{x}(1+U)(1-V)^{-1} d h
$$

exists on $[a, b]$ and, if $f \in S$ and $x \in[a, b]$, then $f(x) \leqslant g(x)$.

Since $Q \in O A^{\circ}$ and $O B^{\circ}$, then

$$
\begin{aligned}
g(x) & =h(x)+(L R) \int_{a}^{x}(g U+g V) \quad \text { (Lemma 5) } \\
& =h(x)+(L I R) \int_{a}^{x}(g P+g Q+g T) \quad \text { (Theorem 3) } \\
& =h(x)+(L I R) \int_{a}^{x}(g k H+g k G+g k K)
\end{aligned}
$$

for $x \in[a, b]$. Therefore, $g \in S$.

In a recent paper [14, Theorem 2.1] Wright, Klasi and Kennebeck consider a Gronwall inequality of the form

$$
f(x) \leqslant \epsilon+(L I R) \int_{a}^{x}\left(w_{1} f k d g+w_{2} f k d g+w_{3} f k d g\right),
$$

where $g$ is nondecreasing and $f$ and $k$ are bounded nonnegative functions on $[a, b]$, $\epsilon$ is a positive number, and $m=\operatorname{lub}_{x \in[a, b]} k(x)$; also,

$$
\begin{gathered}
\alpha(t)=\left|w_{2}+w_{3}\right| m\left[g\left(t^{+}\right)-g(t)\right]<1 \text { and } \\
\beta(t)=\left|w_{3}\right| m\left[g(t)-g\left(t^{-}\right)\right]<1 .
\end{gathered}
$$

Theorem 2.1 is a special case of Theorem 5, Conclusion 1 , with $h=\epsilon, H=w_{1} d g$, $G=w_{2} d g$ and $K=w_{3} d g$. Following is the outline of a proof which shows that there is a positive number $c$ such that $1-m\left(G^{+}+K\right) \geqslant c>0$ on $[a, b]$. Suppose $D=\left\{t_{i}\right\}_{0}^{n}$ is a subdivision of $[a, b]$ and that $0<i \leqslant n$. Then

$$
\begin{aligned}
& m\left(G_{i}^{+}+K_{i}\right)=m w_{2}\left[g\left(t_{i-1}^{+}\right)-g\left(t_{i-1}\right)\right]+m w_{3}\left[g\left(t_{i}\right)-g\left(t_{i-1}\right)\right] \\
& \leqslant m\left|w_{2}+w_{3}\right|\left[g\left(t_{i-1}^{+}\right)-g\left(t_{i-1}\right)\right]+m\left|w_{3}\right|\left[g\left(t_{i}\right)-g\left(t_{i}^{-}\right)\right] \\
&+m\left|w_{3}\right|\left[g\left(t_{i}^{-}\right)-g\left(t_{i-1}^{+}\right)\right] \\
&=\alpha\left(t_{i-1}\right)+\beta\left(t_{i}\right)+m\left|w_{3}\right|\left[g\left(t_{i}^{-}\right)-g\left(t_{i-1}^{+}\right)\right] .
\end{aligned}
$$

Since $g$ is nondecreasing, inequalities (1) and (2) above assure that there is a positive number $c$ such that $1>\alpha(t)+4 c$ and $1>\beta(t)+4 c$ on $[a, b]$; also, if the subdivision $D$ is chosen properly, then $\alpha\left(t_{i-1}\right)+\beta\left(t_{i}\right)<(1-4 c)+c$ and 


$$
m\left|w_{3}\right|\left[g\left(t_{i}^{-}\right)-g\left(t_{i-1}^{+}\right)\right]<c
$$

hence, $m\left(G_{i}^{+}+K_{i}\right)+c<1$. Therefore, any set of functions and numbers which satisfies the hypothesis of [14, Theorem 2.1] will also satisfy the hypothesis of Theorem 5, Conclusion 1.

Lemmas. The following lemmas were used in the proofs of the preceding theorems.

LEMMA 1. If $G$ is a function from $R \times R$ to $R$ such that $\int_{a}^{b} G$ exists, then $G \in O A^{\circ}$ on $[a, b] \quad[3$, Theorem 4.1, p. 304].

LEMMA 2. If $H$ and $G$ are functions from $R \times R$ to $R$ such that $H \in O L^{\circ}$ and $G \in O A^{\circ}$ and $O B^{\circ}$ on $[a, b]$, then $G H \in O A^{\circ}$ and $O M^{\circ}$ on $[a, b] \quad[4$, Theorem 2, p. 494].

LEMMA 3. Given. $[a, b]$ is a number interval, $f$ and $g$ are functions from $R$ to $R$ such that $A \in O B^{\circ}$., the following integrals exist and $\int_{x}^{y} A=\int_{x}^{y}\left(A^{+}+A^{-}\right)$ for $a \leqslant x<y \leqslant b$.

Conclusion. If $\epsilon>0$, then there is a subdivision $D$ of $[a, b]$ such that, if $D^{\prime}=\left\{x_{i}\right\}_{0}^{n}$ is a refinement of $D$ and $\left\{t_{i}\right\}_{1}^{n}$ is an interpolating sequence for $D^{\prime}$ and $P$ is the subset of $\{1,2, \ldots, n\}$ such that $i \in P$ iff $x_{i-1} \notin D$, and $x_{i} \notin D$, then

$$
\sum_{i \in P}\left|\left[f\left(x_{i}\right)-f\left(t_{i}\right)\right]\left[g\left(t_{i}\right)-g\left(x_{i-1}\right)\right]\right|<\epsilon
$$

and

$$
\sum_{i \in P}\left|\left[f\left(t_{i}\right)-f\left(x_{i-1}\right)\right]\left[g\left(x_{i}\right)-g\left(t_{i}\right)\right]\right|<\epsilon
$$

Proof. Let $\epsilon>0$. Since $A \in O A^{\circ}$ and $A \in O B^{\circ}$ and

$$
\int_{x}^{y} A=\int_{x}^{y}\left(A^{+}+A^{-}\right)=\sum_{p \in[x, y]}\left[A\left(p^{-}, p\right)+A\left(p, p^{+}\right)\right]
$$

for $a \leqslant x<y \leqslant b$, then there is a subdivision $D$ of $[a, b]$ such that, if $D^{\prime}=$ $\left\{x_{i}\right\}_{0}^{n}$ is a refinement of $D$ and $P$ is the subset of $\{1,2, \ldots, n\}$ such that $i \in P$ iff $x_{i-1} \notin D$ and $x_{i} \notin D$, then

$$
\begin{gathered}
\sum_{1}^{n}\left|A\left(x_{i-1}, x_{i}\right)-\int_{x_{i-1}}^{x_{i}} A\right|<\epsilon / 6, \\
\sum_{i \in P}\left|\int_{x_{i-1}}^{x_{i}}\left(A^{+}+A^{-}\right)\right|<\epsilon / 6,
\end{gathered}
$$

and 
(3)

$$
\begin{aligned}
\sum_{i \in P}\left|A\left(x_{i-1}, x_{i}\right)\right| \leqslant & \sum_{i \in P}\left|A\left(x_{i-1}, x_{i}\right)-\int_{x_{i-1}}^{x_{i}} A\right|+\sum_{i \in P}\left|\int_{x_{i-1}}^{x_{i}} A\right| \\
& <\epsilon / 6+\sum_{i \in P}\left|\int_{x_{i-1}}^{x_{i}}\left(A^{+}+A^{-}\right)\right|<\epsilon / 3 .
\end{aligned}
$$

Suppose that $D^{\prime}=\left\{x_{i}\right\}_{0}^{n}$ is a refinement of $D$ and $\left\{t_{i}\right\}_{1}^{n}$ is an interpolating sequence for $D^{\prime}$ and $\left\{r_{i}\right\}_{1}^{n}$ is the sequence of numbers such that

$$
\begin{aligned}
r_{i} & =\left[f\left(t_{i}\right)-f\left(x_{i-1}\right)\right]\left[g\left(x_{i}\right)-g\left(t_{i}\right)\right]+\left[f\left(x_{i}\right)-f\left(t_{i}\right)\right]\left[g\left(t_{i}\right)-g\left(x_{i-1}\right)\right] \\
& =A\left(x_{i-1}, x_{i}\right)-\left[A\left(x_{i-1}, t_{i}\right)+A\left(t_{i}, x_{i}\right)\right]
\end{aligned}
$$

for $i=1,2, \ldots, n$. It follows from inequality (1) that

$$
\begin{aligned}
\sum_{1}^{n}\left|r_{i}\right| \leqslant \sum_{1}^{n}\left[\left|A\left(x_{i-1}, x_{i}\right)-\int_{x_{i-1}}^{x_{i}} A\right|+\left|\int_{x_{i-1}}^{t_{i}} A-A\left(x_{i-1}, t_{i}\right)\right|\right. \\
\left.+\left|\int_{t_{i}}^{x_{i}} A-A\left(t_{i}, x_{i}\right)\right|\right]<\epsilon / 2 .
\end{aligned}
$$

We will now prove the inequalities in the conclusion. Let $P$ be the subset of $\{1,2, \ldots, n\}$ such that $i \in P$ iff $x_{i-1} \notin D$ and $x_{i} \notin D$, and let $\left\{a_{i}\right\}_{1}^{n}$ and $\left\{b_{i}\right\}_{1}^{n}$ be the number sequences such that, if $0<i \leqslant n$, then:

(1) if $\left|g\left(x_{i}\right)-g\left(t_{i}\right)\right|>\left|g\left(t_{i}\right)-g\left(x_{i-1}\right)\right|$, then $a_{i}=1$ and $b_{i}=0$; and

(2) if $\left|g\left(x_{i}\right)-g\left(t_{i}\right)\right| \leqslant\left|g\left(t_{i}\right)-g\left(x_{i-1}\right)\right|$, then $a_{i}=0$ and $b_{i}=1$.

It follows that

$$
\begin{aligned}
& \begin{array}{l}
\sum_{i \in P}\left|\left[f\left(x_{i}\right)-f\left(t_{i}\right)\right]\left[g\left(t_{i}\right)-g\left(x_{i-1}\right)\right]\right| \\
=\sum_{i \in P}\left\{a_{i}\left|\left[f\left(x_{i}\right)-f\left(t_{i}\right)\right]\left[g\left(t_{i}\right)-g\left(x_{i-1}\right)\right]\right|\right. \\
\left.\quad+b_{i}\left|r_{i}-\left[f\left(t_{i}\right)-f\left(x_{i-1}\right)\right]\left[g\left(x_{i}\right)-g\left(t_{i}\right)\right]\right|\right\} \\
\leqslant \sum_{i \in P}\left\{a_{i}\left|f\left(x_{i}\right)-f\left(t_{i}\right)\right|\left|g\left(x_{i}\right)-g\left(t_{i}\right)\right|\right. \\
\left.\quad+b_{i}\left|f\left(t_{i}\right)-f\left(x_{i-1}\right)\right|\left|g\left(t_{i}\right)-g\left(x_{i-1}\right)\right|+\left|r_{i}\right|\right\}
\end{array} \\
& \leqslant \sum_{i \in P}\left[\left|A\left(t_{i}, x_{i}\right)\right|+\left|A\left(x_{i-1}, t_{i}\right)\right|\right]+\epsilon / 2<\epsilon / 2+\epsilon / 2,
\end{aligned}
$$

from inequality (3), because $D^{\prime} \cup\left\{t_{i}\right\}_{1}^{n}$ is a refinement of $D$. Similarly,

$$
\sum_{i \in P}\left|\left[f\left(t_{i}\right)-f\left(x_{i-1}\right)\right]\left[g\left(x_{i}\right)-g\left(t_{i}\right)\right]\right|<\epsilon .
$$

LEMMA 4. Given. $G$ and $H$ are functions from $R \times R$ to $R$ such that $G \in$ $O A^{\circ}$ and $O B^{\circ}$ on $[a, b]$ and $H(x, y)=(1 / 2)[|G(x, y)|+G(x, y)]$ for $a \leqslant x<$ $y \leqslant b$. 
Conclusion. $|G|$ and $H \in O A^{\circ}$ and $O B^{\circ}$ on $[a, b]$.

OUTLINE OF PROOF. Let $g(x)=\int_{a}^{x} G$. If $D=\left\{x_{i}\right\}_{0}^{n}$ is a subdivision of $[a, b]$, then

$$
\sum_{1}^{n}\left|\Delta g_{i}\right|=\sum_{1}^{n}\left|\int_{x_{i-1}}^{x_{i}} G\right| \leqslant \sum_{1}^{n}\left|G_{i}\right|+\sum_{1}^{n}\left|\int_{x_{i-1}}^{x_{i}} G-G_{i}\right| .
$$

Since $G \in O B^{\circ}$ and $O A^{\circ}$, it follows that $d g \in O B^{\circ}$ and that $\int_{a}^{b}|d g|$ exists. If $\left\{x_{i}\right\}_{0}^{n}$ is a subdivision of $[a, b]$, then

$$
\begin{aligned}
\left|\sum_{1}^{n}\right| \Delta g_{i}\left|-\sum_{1}^{n}\right| G_{i}|| & =\left|\sum_{1}^{n}\left(\left|\int_{x_{i-1}}^{x_{i}} G\right|-\left|G_{i}\right|\right)\right| \\
& \leqslant \sum_{1}^{n}\left|\int_{x_{i-1}}^{x_{i}} G-G_{i}\right| .
\end{aligned}
$$

Since $G \in O A^{\circ}$, the last summation can be made arbitrarily small; it follows that $\int_{a}^{b}|G|$ exists and $|G| \in O A^{\circ}$ (Lemma 1). Since $|G|$ and $G \in O A^{\circ}$ and $O B^{\circ}$, then $H=(1 / 2)(|G|+G) \in O A^{\circ}$ and $O B^{\circ}$.

Lemma 5. Given. $[a, b]$ is a number interval, $c>0$ and $h$ is a function from $R$ to $R$ such that $d h \in O B^{\circ}$ on $[a, b] ; U$ and $V$ are functions from $R \times R$ to $R$ such that $U$ and $V \in O A^{\circ}$ and $O B^{\circ}$ and $1-V \geqslant c>0$ on $[a, b]$.

Conclusion. (1) If $a \leqslant x<y \leqslant b$, then ${ }_{x} \Pi^{y}(1+U)(1-V)^{-1}$ and (R) $\int_{a}^{y}(1-V)^{-1}{ }_{t} \Pi^{y}(1+U)(1-V)^{-1} d h$ exist. (2) If $g$ is the function such that $g(x)=h(a)_{a} \Pi^{x}(1+U)(1-V)^{-1}+(R) \int_{a}^{x}(1-V)^{-1}{ }_{t} \Pi^{x}(1+U)(1-V)^{-1} d h$ for $x \in[a, b]$, then $g(x)=h(x)+(L R) \int_{a}^{x}(g U+g V)$ for $x \in[a, b]$.

Proof. We will show that Lemma 5 is a corollary to Theorem 5.1 of [4]. Since $V \in O A^{\circ}$ and $O B^{\circ}$ and $1-V \geqslant c>0$ on $[a, b]$, then $(1-V)^{-1}$ exists, is bounded, and $\in O L^{\circ}$ on $[a, b]$. Since $(1-V)^{-1} \in O L^{\circ}$ and $d h \in O A^{\circ}$ and $O B^{\circ}$ then $(1-V)^{-1} d h \in O A^{\circ}$ (Lemma 2). Since $(1-V)^{-1} \in O L^{\circ}$ and is bounded, since $U+V \in O B^{\circ}$ and $O A^{\circ}$, and since $(1+U)(1-V)^{-1}-1=(U+V)$. $(1-V)^{-1}$, then $(1+U)(1-V)^{-1}-1 \in O B^{\circ}, O A^{\circ}$ and $O M^{\circ}$ (Lemma 2); hence ${ }_{x} \Pi^{y}(1+U)(1-V)^{-1}$ exists for $a \leqslant x<y \leqslant b$. Since the function $r(t)=$ ${ }_{t} \Pi^{y}(1+U)(1-V)^{-1}$ has bounded variation and $(1-V)^{-1} d h \in O A^{\circ}$ and $O B^{\circ}$, then

$$
(R) \int_{x}^{y}\left[\Pi_{t} \Pi^{y}(1+U)(1-V)^{-1}\right](1-V)^{-1} d h
$$

exists for $a \leqslant x<y \leqslant b$ (Lemma 2). Let $g$ be the function defined in Conclusion 2. Since the functions $U, V, h$ and $g$ satisfy the hypothesis of Theorem 5.1, $2 \rightarrow 1$, it follows that $g(x)+h(x)+(L R) \int_{a}^{x}(g U+g V)$ for $x \in[a, b]$. 
The proof of Lemma 6 is similar to the proof of Theorem 3 of [3].

LEMMA 6. Given. $H$ and $G$ are functions from $R \times R$ to $R$ and $c$ is $a$ positive number such that $H$ and $G \in O A^{\circ}$ and $O B^{\circ}, H \geqslant 0, G \geqslant 0$, and $1-G \geqslant$ $c$ on $[a, b] ; u$ is a function from $R$ to $R$ such that $u$ is bounded above on $[a, b]$, $u(a) \leqslant 0$ and, if $\epsilon>0$ and $a<p \leqslant b$, then there is a subdivision $D$ of $[a, p]$ such that if $\left\{x_{i}\right\}_{0}^{n}$ is a refinement of $D$ then

$$
u\left(x_{n}\right) \leqslant \sum_{1}^{n}\left[u\left(x_{i-1}\right) H\left(x_{i-1}, x_{i}\right)+u\left(x_{i}\right) G\left(x_{i-1}, x_{i}\right)\right]+\epsilon .
$$

Conclusion. If $x \in[a, b]$, then $u(x) \leqslant 0$.

Proof. Assume the conclusion is false, and let $S$ be the subset of $[a, b]$ such that $x \in S$ iff $u(x)>0$; then $S$ is nonempty and has a greatest lower bound $p$.

Suppose that $p \in S$; then $u(p)>0$ and $p \neq a$. Since $1-G \geqslant c>0$, there is a positive number $k$ such that $2 k<u(p)\left[1-G\left(p^{-}, p\right)\right]$. Since $G \in O B^{\circ}$ and $O A^{\circ}$ and since $k>0$, there is a subdivision $\left\{x_{i}\right\}_{0}^{n}$ of $[a, p]$ such that

$$
u(p) G\left(x_{n-1}, p\right)-u(p) G\left(p^{-}, p\right)<k
$$

and

$$
\begin{aligned}
u(p) & \leqslant \sum_{1}^{n}\left[u\left(x_{i-1}\right) H\left(x_{i-1}, x_{i}\right)+u\left(x_{i}\right) G\left(x_{i-1}, x_{i}\right)\right]+k \leqslant u(p) G\left(x_{n-1}, p\right)+k \\
& =\left[u(p) G\left(x_{n-1}, p\right)-u(p) G\left(p^{-}, p\right)\right]+u(p) G\left(p^{-}, p\right)+k \\
& \leqslant k+u(p) G\left(p^{-}, p\right)+k
\end{aligned}
$$

therefore, $u(p)\left[1-G\left(p^{-}, p\right)\right] \leqslant 2 k$. Since this contradicts an earlier statement that $2 k<u(p)\left[1-G\left(p^{-}, p\right)\right]$, it follows that $p \notin S$.

Since $p \notin S$, then $u(t) \leqslant 0$ for $a \leqslant t \leqslant p$ and $p \neq b$. Since $G$ and $H \in O B^{\circ}$ and since $1-G\left(p, p^{+}\right) \geqslant c>0$, there is a positive number $k$ such that

$$
(1 / 2)\left[G\left(p, p^{+}\right)+1+k\right]<1 \text {. }
$$

Since $H$ and $G \in O B^{\circ}$, there is a number $y \in(p, b]$ such that, if $\left\{x_{i}\right\}_{0}^{n}$ is a subdivision of $[p, y]$, then

$$
\begin{aligned}
\sum_{2}^{n} H\left(x_{i-1}, x_{i}\right)+\sum_{i}^{n} G\left(x_{i-1}, x_{i}\right) & <(1 / 2)\left[1-G\left(p, p^{+}\right)\right]+G\left(p, p^{+}\right) \\
& =(1 / 2)\left[1+G\left(p, p^{+}\right)\right]<1 .
\end{aligned}
$$

Let $M$ be the least upper bound for $u$ on $[p, y]$; then there is a number $z \in$ $(p, y]$ such that

$$
u(z)>(1 / 2)\left[G\left(p, p^{+}\right)+1+k\right] M
$$


It follows from the hypothesis that there is a subdivision $D=\left\{z_{i}\right\}_{0}^{n}$ of $[a, z]$ and an integer $r$ such that $p=z_{r-1} \in D$ and such that

$$
\begin{aligned}
u(z) & \leqslant \sum_{1}^{n}\left[u\left(z_{i-1}\right) H\left(z_{i-1}, z_{i}\right)+u\left(z_{i}\right) G\left(z_{i-1}, z_{i}\right)\right]+k M / 2 \\
& \leqslant \sum_{r+1}^{n} u_{i-1} H_{i}+\sum_{r}^{n} u_{i} G_{i}+k M / 2 \leqslant\left[\sum_{r+1}^{n} H_{i}+\sum_{r}^{n} G_{i}\right] M+k M / 2 \\
& <(1 / 2)\left[1+G\left(p, p^{+}\right)\right] M+(1 / 2) k M \text { (Inequality 1) } \\
& =(1 / 2)\left[1+G\left(p, p^{+}\right)+k\right] M<u(z) . \text { (Inequality 2). }
\end{aligned}
$$

It follows that the conclusion is true.

\section{BIBLIOGRAPHY}

1. S. C. Chu and F. T. Metcalf, On Gronwall's inequality, Proc. Amer. Math. Soc. 18 (1967), 439-440. MR 35 \#3400.

2. Ben Dushnik, On the Stieltjes integral, Dissertation, University of Michigan, 1931.

3. B. W. Helton, Integral equations and product integrals, Pacific J. Math. 16 (1966), 297-322. MR 32 \#6167.

4. B. W. Helton, A product integral representation for a Gronwall inequality, Proc. Amer. Math. Soc. 23 (1969), 493-500. MR 40 \#1562.

5. - The solution of a nonlinear Gronwall inequality, Proc. Amer. Math. Soc. 38 (1973), 337-342. MR 46 \#9287.

6. J. V. Herod, A Gronwall inequality for linear Stieltjes integrals, Proc. Amer. Math. Soc. 23 (1969), 34-36. MR 40 \#2802.

7. J. R. Kroll and K. P. Smith, An eigenvalue problem for the Stieltjes mean sigmaintegral related to Gronwall's inequality, Proc. Amer. Math. Soc. 33 (1972), 384-388. MR 45 \#833.

8. W. W. Schmaedeke and G. R. Sell, The Gronwall inequality for modified Stieltjes integrals, Proc. Amer. Math. Soc. 19 (1968), 1217-1222. MR 37 \#6422.

9. H. L. Smith, On the existence of the Stieltjes integral, Trans. Amer. Math. Soc. 27 (1925), 491-515.

10. D. R. Snow, Gronwall's inequality for systems of partial differential equations in two independent variables, Proc. Amer. Math. Soc. 33 (1972), 46-54. MR 45 \#7240.

11. D. W. Willett, A linear generalization of Gronwall's inequality, Proc. Amer. Math. Soc. 16 (1965), 774-778. MR 31 \#5953.

12. D. W. Willett and J. S. W. Wong, On the discrete analogues of some generalizations of Gronwall's inequality, Monatsh. Math. 69 (1965), 362-367. MR 32 \#2644.

13. F. M. Wright and J. D. Baker, On integration-by-parts for weighted integrals, Proc. Amer. Math. Soc. 22 (1969), 42-52. MR 39 \#7056.

14. F. M. Wright, M. L. Klasi and D. R. Kennebeck, The Gronwall inequality for weighted integrals, Proc. Amer. Math. Soc. 30 (1971), 504-510. MR 44 \#380.

15. Yue-sheng Li, The bound, stability and error estimates for the solution of nonlinear differential equations, Acta Math. Sinica 12 (1962), 32-39 = Chinese Math. 3 (1963), 34-41. MR 27 \#405.

DEPARTMENT OF MATHEMATICS, SOUTHWEST TEXAS STATE UNIVERSITY, SAN MARCOS, TEXAS 78666 